Atıf/Citation: E. KARAMAN, “Gömme Fonksiyonu Kullanılarak Küme Optimizasyonuna Göre Verilen Küme Değerli Optimizasyon Problemlerinin Optimallik Koşulları”, Süleyman Demirel Üniversitesi Fen Edebiyat Fakültesi Fen Dergisi, 14, 105-111, 2019.

\title{
Gömme Fonksiyonu Kullanılarak Küme Optimizasyonuna Göre Verilen Küme Değerli Optimizasyon Problemlerinin Optimallik Koşulları
}

\author{
Emrah KARAMAN
}

\author{
Karabük Üniversitesi, Fen Fakültesi, Matematik Bölümü, 78050, Karabük, Türkiye \\ *yazışllan yazar e-posta: e.karaman42@gmail.com
}

(Alınlş / Received: 10.11.2018, Kabul / Accepted: 28.03.2019, Yaylmlanma / Published: 31.05.2019)

Özet: $\mathrm{Bu}$ çalışmada küme değerli optimizasyon problemi ele alınmıştır. Küme değerli optimizasyon problemlerinin çözümlerini bulmak için kullanılan bazı kriterler vardır. Literatürde en çok kullanılan kriterler küme yaklaşımı ve vektör yaklaşımıdır. Bu çalışmada ise küme değerli optimizasyon problemlerinin küme yaklaşımına göre çözümleri araştırıldı. Küme değerli optimizasyon problemlerinin küme yaklaşımına göre optimallik koşullarını elde etmek için skalerizasyon, vektörizasyon ve yönlü türev gibi yöntemler kullanılır. Bu yöntemlerden farklı olarak çalışmada problemin optimallik koşullarını elde etmek için gömme fonksiyonu kullanılmıştır. Gömme fonksiyonu ile optimallik koşullarını elde edebilmek için bir gömme uzayı kullanılmıştır. Bu uzayın ve gömme fonksiyonunun bazı özellikleri incelenmiştir. Bunlara ek olarak çalışmanın daha iyi anlaşılabilmesi için bir örnek verilmiştir.

Anahtar kelimeler: Küme değerli dönüşüm, optimallik koşulu, gömme fonksiyonu.

\section{Optimality Conditions of Given Set-valued Optimization Problems with Respect to Set Optimization by Using Embedding Function}

\begin{abstract}
In this current study, set-valued optimization problem is considered. There are some criteria to obtain solutions of this set-valued optimization problem. The two most commonly used criteria are set and vector approaches in the literature. In this work, we investigated the solutions of set-valued optimization problems with respect to set approach. Many methods such as scalarization, vectorization and directional derivative are used to find the optimality conditions of set-valued optimization problems with respect to set approach. Apart from these methods, we used embedding function to obtain optimality conditions in this study. An embedding space is used in order to obtain optimality conditions by using embedding function. Some properties of this space and embedding function are studied. Moreover, an example is given to understand more better of the study.
\end{abstract}

Keywords: Set-valued map, optimality condition, embedding function.

\section{Giriş}

Küme değerli dönüşümlerin optimizasyonu son zamanlarsa çok ilgi görmektedir. Bunun sebeplerinden bazıları problemin finans, kontrol teori, oyun teori, mühendislik gibi alanlarda uygulamasının olmasıdır [1, 2]. Küme değerli optimizasyon problemlerini çözebilmek için kullanılan bazı yaklaşımlar vardır. Literatürde en çok kullanılanlar vektör ve küme yaklaşımıdır [2, 3, 4, 5]. Araştırmacıların ilk kullandıkları çözüm kriteri vektör yaklaşımıdır. Küme değerli optimizasyon problemini vektör yaklaşımına göre çözebilmek için görüntü kümesi üzerinde işlem yapmak gerekmektedir. Bir noktanın problemin çözümü olabilmesi için o noktanın küme değerli dönüşüm altındaki 
görüntüsü küme değerli dönüşümün görüntü kümesinin en az bir etkin (minimal veya maksimal) noktasını içermelidir.

Yakın bir zamanda Kuroiwa tarafından ortaya çıkarılan küme yaklaşımı ise küme değerli dönüşümün görüntü kümeleri arasındaki karşılaştırmaya dayanmaktadır. $\mathrm{Bu}$ yüzden problemi küme yaklaşımına göre çözebilmek için kümeler arasında sıralama bağıntısına ihtiyaç vardır. Bu sıralama bağıntısına göre küme değerli dönüşümün görüntü kümeleri arasında etkin kümeler bulunur. Etkin kümelerin ters görüntüsü problemin küme yaklaşımına göre çözümünü verir.

Sıralama konisine bağlı olarak küme sıralamaları hakkında ilk çalışma Kuroiwa vd. [6] tarafından yapılmıştır. Yaptıkları çalışmada altı tane sıralama bağıntı tanımlamışlardır. Daha sonra Kuroiwa bu altı sıralama bağıntısını kullanarak küme yaklaşımı teorisini ortaya çıkarmıştır [7, 8]. Yaptığı çalışmada bu sıralama bağıntılarına göre bir kümeler ailesinin minimal ve maksimal kümelerini de tanımlamıştır. Daha sonra, Jahn ve Ha [9] küme değerli optimizasyonda kullanmak üzere yeni sıralama bağıntıları tanımlamıştır. Literatürde kullanılan sıralama bağıntılarının bazıları ön sıralama bağıntısı bazıları da ön sıralama bağıntısı bile değildir. Literatürdeki ilk kısmi sıralama bağıntısı Karaman vd. [4] tarafından tanımlanmıştır. Minkowski fark kullanarak tanımlanan bu sıralama bağıntısının sınırlı kümeler ailesi üzerinde bir kısmi sıralama bağıntısı olduğu gösterilmiştir. Aynı çalışmada kısmi sıralama bağıntısına göre verilen küme değerli optimizasyon problemlerinin optimallik koşullarını elde edebilmek için skalerizasyon yöntemi de elde edilmiştir.

Küme değerli optimizasyon problemlerini küme yaklaşımına göre çözebilmek için kullanılan birçok yöntem vardır. Küme değerli optimizasyon problemi skaler optimizasyon problemi ile karakterize edildiğinde elde edilen yöntem skalerizasyon [5], küme değerli optimizasyon problemi vektör optimizasyon problemi ile karakterize edildiğinde elde edilen yöntem vektörizasyon [3] olarak adlandırılmaktadır. Küme değerli optimizasyon problemlerinin optimallik koşullarını elde etmek için yönlü türevlerden de yararlanılmaktadır $[10,11]$. Problemin optimallik koşullarını elde etmek için kullanılan bir başka yöntem ise gömme dönüşümüdür. $\mathrm{Bu}$ yöntem literatürde sadece Kuroiwa tarafından kullanılmıştır [11, 12, 13, 14].

$\mathrm{Bu}$ çalışmada, küme değerli optimizasyon problemlerinin optimallik koşullarını elde etmek için Kuroiwa tarafından kullanılan gömme fonksiyonu ele alınmıştır. Gömme fonksiyonunu tanımlayabilmek ve üzerinde işlemler yapabilmek için Kuroiwa [11]'nın kullandığı gömme uzayı kullanılmıştır. Gömme uzayı ve gömme fonksiyonunun bazı özellikleri incelendikten sonra küme yaklaşımına göre verilen küme değerli optimizasyon problemlerinin optimallik koşulları elde edilmiştir. Son olarak, elde edilen optimallik koşulları bir örnek üzerinde incelenmiştir.

\section{Materyal ve Metot}

Bu çalışmada, $X$ boştan farklı bir küme, $Y$, kapalı, konveks, sivri $\left(K \cap(-K)=\left\{0_{Y}\right\}\right)$ ve içi boştan farklı bir $K \subset Y$ konisi (her $x \in K$ ve her $\lambda>0$ için $\lambda x \in K$ ) ile kısmi sıralı normlu vektör uzayı olarak alınacaktır. $Y$ 'nin boştan farklı tüm alt kümelerinin ailesi $\mathcal{P}(Y)$ ile gösterilecektir. Yani

$$
\mathcal{P}(Y):=\{A \subset Y \mid A \neq \emptyset\}^{\prime} \text { dir. }
$$

$A, B \in \mathcal{P}(Y)$ kümesi verildiğinde, $A$ kümenin içini $\operatorname{int}(A), A$ ve $B$ kümelerinin konveks zarfi yani $A$ ve $B$ kümelerini bulunduran tüm konveks kümelerin kesişimi 
$\operatorname{conv}\{A, B\}$ ile gösterilecektir. $Y$ 'nin boştan farklı kompakt, konveks alt kümelerinin ailesi $\mathcal{C}(Y)$ ile gösterilsin. $\mathrm{O}$ halde

$$
\mathcal{C}(Y):=\{A \subset Y \mid \text { A kompakt, konveks }\} \text { 'dir. }
$$

$K$ konisi yardımıyla $Y$ üzerinde bir kısmi sıralama bağıntısı; $\forall x, y \in Y$ için

$$
\begin{gathered}
x \leq_{K} y \Leftrightarrow y-x \in K \\
x<_{K} y \Leftrightarrow y-x \in \operatorname{int}(K)
\end{gathered}
$$

şeklinde tanımlanır.

$A \in \mathcal{P}(Y)$ ve $x_{0} \in Y$ olsun. $A \cap\left(x_{0}-K\right)=\left\{x_{0}\right\}\left(A \cap\left(x_{0}+K\right)=\left\{x_{0}\right\}\right)$ ise $x_{0}$ 'a $A$ kümesinin $K$ konisine göre minimal (maksimal) elemanı denir. $A \cap\left(x_{0}-\operatorname{int}(K)\right)=\varnothing$ $\left(A \cap\left(x_{0}+\operatorname{int}(K)\right)=\emptyset\right)$ ise $x_{0}$ 'a $A$ kümesinin $K$ konisine göre zayıf minimal (zayıf maksimal) elemanı denir.

Tanım 2.1. $A, B \in \mathcal{P}(Y)$ olsun.

i. $\quad A+B=\{a+b \mid a \in A$ ve $b \in B\}$ kümesine $A$ ve $B$ kümelerinin cebirsel toplami,

ii. $\quad A-B=\{a-b \mid a \in A$ ve $b \in B\}$ kümesine $A$ ve $B$ kümelerinin cebirsel fark1,

iii. $\quad A-B=\{x \mid x+B \subset A\}$ kümesine $A$ ve $B$ kümelerinin Minkowski (Pontryagin) fark1 denir.

Kümelerin Minkowski farkı aşağıdaki özelliklere sahiptir. Bu konu ile ilgili daha fazla bilgi $[4,15,16]$ çalışmalarında bulunabilir.

Önerme 2.2. [4] $A, B \in \mathcal{P}(Y)$ ve $x \in Y$ olsun.

i. $\quad(x+A)-B=x+(A-B)$,

ii. $\quad A-(x+B)=-x+(A-B)$,

iii. $\quad A$ kapalı ise $A-B$ kapalı,

iv. $\quad A$ sinurlı ise $A-A=\left\{0_{Y}\right\}^{\prime}$ dir.

$F: X \rightrightarrows Y$ bir küme değerli dönüşüm olsun. $N, Y$ üzerinde herhangi bir kümesel özellik olmak üzere, $\forall x \in X$ için $F(x)$ kümeleri $Y$ üzerinde $N$ özelliğini sağlıyorsa $F$ 'ye $N$ değerli denir. Küme değerli optimizasyon problemi

$$
(S O P)\left\{\begin{array}{c}
\min (\operatorname{maks}) F(x) \\
x \in X
\end{array}\right.
$$

şeklinde tanımlanır. (SOP)'nin çözümlerinin elde edilmesinde kullanılan yaklaşımlardan biri küme yaklaşımıdır. Bu yaklaşım $F$ 'nin değerleri olan kümeler arasındaki karşılaştırmaya dayanmaktadır. Bu karşılaştırmayı yapabilmek için $\mathcal{P}(Y)$ üzerinde sıralama bağıntısına ihtiyaç vardır. Sınırlı kümeler ailesi üzerindeki ilk kısmi sıralama bağıntısı aşağıda verilmiştir.

Tanım 2.3. [4] $A, B \in \mathcal{P}(Y)$ olsun. $\mathcal{P}(Y)$ üzerinde $m_{1}$ ve kesin $m_{1}$ bağıntıları sırasıyla

ve

$$
A \preccurlyeq_{K}^{m_{1}} B: \Leftrightarrow(B \dot{-A}) \cap K \neq \varnothing
$$

$$
A \prec_{K}^{m_{1}} B: \Leftrightarrow(B \dot{-A}) \cap \operatorname{int}(K) \neq \emptyset
$$


şeklinde tanımlanır.

$A$ ve $B$ kümelerini tek nokta kümesi olarak aldığımızda $\preccurlyeq_{K}^{m_{1}}$ bağıntısı $Y$ üzerindeki $\leq_{K}$ bağıntısına indirgenir. Bu yüzden $\preccurlyeq_{K}^{m_{1}}$ bağıntısı $Y$ üzerinde bilinen $\leq_{K}$ bağıntısının kümeler ailesi üzerine bir genişlemesidir. $\preccurlyeq_{K}^{m_{1}}$ bağıntısı sınırlı kümeler ailesi üzerinde bir kısmi sıralama bağıntısıdır [4]. Bu bağıntıya göre bir kümeler ailesinin minimal, maksimal, zayıf minimal ve zayıf maksimal elemanları aşağıdaki şekilde tanımlanır.

Tanım 2.4. [4] $\mathcal{S} \subset \mathcal{P}(Y)$ ve $A \in \mathcal{S}$ olsun.

i. $\quad B \preccurlyeq_{K}^{m_{1}} A$ ve $A \neq B$ olacak şekilde bir $B \in \mathcal{S}$ kümesi yoksa $A$ 'ya $\mathcal{S}$ ailesinin bir $m_{1}$-minimal eleman1,

ii. $\quad A \preccurlyeq_{K}^{m_{1}} B$ ve $A \neq B$ olacak şekilde bir $B \in \mathcal{S}$ kümesi yoksa $A$ 'ya $\mathcal{S}$ ailesinin bir $m_{1}$-maksimal elemanı,

iii. $\quad B \prec_{K}^{m_{1}} A$ ve $A \neq B$ olacak şekilde bir $B \in \mathcal{S}$ kümesi yoksa $A$ 'ya $\mathcal{S}$ ailesinin bir zayıf $m_{1}$-minimal elemanı,

iv. $\quad A \prec_{K}^{m_{1}} B$ ve $A \neq B$ olacak şekilde bir $B \in \mathcal{S}$ kümesi yoksa $A$ 'ya $\mathcal{S}$ ailesinin bir zayıf $m_{1}$-maksimal elemanı denir.

$(S O P), \preccurlyeq_{K}^{m_{1}}$ bağıntısı kullanarak küme yaklaşımına göre incelediğinde problem $\left(m_{1}-\right.$ $S O P$ ) ile gösterilir. $\mathcal{F}(X):=\{F(x) \mid x \in X\}$ olmak üzere $F\left(x_{0}\right), \mathcal{F}(X)$ ailesinin bir $m_{1}$ minimal $\left(m_{1}\right.$-maksimal) kümesi ise $x_{0}$ 'a $\left(m_{1}-S O P\right)$ 'nin bir çözümü denir. Benzer olarak $\left(m_{1}-S O P\right)$ verildiğinde $F\left(x_{0}\right), \mathcal{F}(X)$ ailesinin bir zayıf $m_{1}$-minimal (zayıf $m_{1}$ maksimal) kümesi ise $x_{0}$ 'a $\left(m_{1}-S O P\right)$ 'nin bir zayıf çözümü denir.

\section{Bulgular}

$\mathcal{C}(Y)^{2}$, de $\equiv$ ikili bağıntısı $\forall(A, B),(C, D) \in \mathcal{C}(Y)^{2}$ için

$$
(A, B) \equiv(C, D): \Leftrightarrow A+D+K=B+C+K
$$

şeklinde tanımlansın. $\equiv$ bağıntısı $\mathcal{C}(Y)^{2}$ üzerinde bir denklik bağıntısıdır. Bunu gösterebilmek için literatürde Cancellation law olarak bilinen $A+B=C+B \Rightarrow A=C$ sadeleştirmesi kullanılır $[14,15] .(A, B)$ 'nin denklik sınıflarını $[A, B]$ ile gösterelim. $\mathrm{O}$ halde

$$
[A, B]:=\left\{(C, D) \in \mathcal{C}(Y)^{2} \mid(A, B) \equiv(C, D)\right\}
$$

olur. $\equiv$ denklik bağıntısının $\mathcal{C}(Y)^{2}$ üzerindeki gömme uzayı $M$ ile gösterilsin. O zaman $M=\mathcal{C}(Y)^{2} / \equiv:=\left\{[A, B] \mid(A, B) \in \mathcal{C}(Y)^{2}\right\}^{\prime}$ dir. Gömme uzayı üzerinde toplama ve skaler ile çarpma işlemi $\forall[A, B],[C, D] \in M$ ve $\forall \alpha \in \mathbb{R}$ için

$$
\begin{gathered}
{[A, B]+[C, D]=[A+C, B+D],} \\
\alpha \cdot[A, B]=\left\{\begin{array}{c}
{[\alpha A, \alpha B], \quad \alpha \geq 0} \\
{[-\alpha B,-\alpha A], \quad \alpha<0}
\end{array}\right.
\end{gathered}
$$

olduğunda $(M,+,) \mathbb{R}$ üzerinde bir vektör uzayıdır. Bu uzayın etkisiz elemanı ise $\left[\left\{0_{Y}\right\},\left\{0_{Y}\right\}\right]^{\prime}$ dir.

M'nin özel bir alt kümesi

$$
\mathcal{K}:=\left\{[A, B] \in M \mid B \preccurlyeq_{K}^{m_{1}} A\right\}
$$


olsun. $\mathcal{K}, M^{\prime}$ 'de konveks, sivri bir konidir. $M^{\prime}$ de $\mathcal{K}$ kümesiyle $\preccurlyeq_{\mathcal{K}}$ sıralama bağıntısı

$$
[A, B] \preccurlyeq_{\mathcal{K}}[C, D]: \Leftrightarrow[C, D]-[A, B] \in \mathcal{K}
$$

şeklinde tanımlansın. Bu sıralama bağıntısı $M$ üzerinde bir kısmi sıralama bağıntısıdır. $M$ üzerinde kesin küçük bağıntısını tanımlamak için $\operatorname{int}(\mathcal{K})$ kümesini de

$$
\operatorname{int}(\mathcal{K}):=\left\{[A, B] \in M \mid B \prec_{\mathcal{K}} A\right\}
$$

şeklinde tanımlayalım. Bu durumda $\prec_{\mathcal{K}}$ bağıntısı

$$
[A, B] \prec_{\mathcal{K}}[C, D]: \Leftrightarrow[C, D]-[A, B] \in \operatorname{int}(\mathcal{K})
$$

olur.

$K$ 'nın bir tabanı $S$ olsun. O halde $K=\bigcup_{\lambda \geq 0} \lambda S$ 'dir. $M$ üzerinde $\|\cdot\|: M \rightarrow \mathbb{R}$ normu her $[A, B] \in M$ için

$$
\|[A, B]\|=\sup _{s \in S}|\inf \langle s, A\rangle-\inf \langle s, B\rangle|
$$

şeklinde tanımlandığında $M$ normlu vektör uzayı olur.

$\left(m_{1}-S O P\right)$ 'yi karakterize etmek için kullanacağımız $T: \mathcal{C}(Y) \rightarrow M$ gömme fonksiyonunu $\forall A \in \mathcal{C}(Y)$ için

$$
T(A)=[A,\{0\}]
$$

şeklinde tanımlayalım. $T$ fonksiyonu yardımıyla $\left(m_{1}-S O P\right)$ için optimallik koşullarını elde etmek için fonksiyonun aşağıda verilen özelliklerine ihtiyaç vardır.

Önerme 3.1. $A, B \in \mathcal{C}(Y)$ olsun. $A \preccurlyeq_{K}^{m_{1}} B$ olması için gerek ve yeter koşul $T(A) \preccurlyeq_{\mathcal{K}} T(B)$ olmasidir.

Kanit: Kabul edelim ki $A \preccurlyeq_{K}^{m_{1}} B$ olsun. Bu durumda $[B, A]=[B,\{0\}]-[\{0\}, A] \in \mathcal{K}$ olur. $T$ fonksiyonunun tanımından $T(B)-T(A) \in \mathcal{K}$ yani $T(A) \preccurlyeq_{\mathcal{K}} T(B)$ elde edilir.

Ters kapsam da benzer şekilde gösterilir.

Önerme 3.2. $A, B \in \mathcal{C}(Y)$ olsun. $A \prec_{K}^{m_{1}} B$ olması için gerek ve yeter koşul $T(A) \prec_{\mathcal{K}} T(B)$ olmasidir.

Kanıt: Önerme 3.1.'in kanıtına benzer şekilde elde edilir.

Teorem 3.3. ( $\left.m_{1}-S O P\right)^{\prime}$ 'nin optimallik koşullarını elde etmemize olanak sağlayacaktır.

Teorem 3.3. $F: X \rightrightarrows Y$ kompakt, konveks değerli bir dönüşüm olsun. $x_{0} \in X$ 'in $\left(m_{1}-\right.$ $S O P$ )'nin bir çözümü olması için gerek ve yeter koşul $x_{0}$ '’n

$$
(E-S O P)\left\{\begin{array}{c}
\min (m a k s) T(F(x)) \\
x \in X
\end{array}\right.
$$


probleminin bir çözümü olmasıdır.

Kanıt: Kabul edelim ki $x_{0},\left(m_{1}-S O P\right)$ 'nin bir çözümü olsun. $\mathrm{O}$ halde $\forall x \in X /\left\{x_{0}\right\}$ için $F(x) \preccurlyeq_{K}^{m_{1}} F\left(x_{0}\right)$ olacak şekilde bir $x$ yoktur. Önerme 3.1.'den $\forall x \in X$ için $T(F(x)) \preccurlyeq_{K}^{m_{1}} T\left(F\left(x_{0}\right)\right)$ olacak şekilde bir $x$ 'in olmadı ğını elde ederiz. Bu ise $x_{0}$ ' $(E-S O P)^{\prime}$ 'nin çözümü olduğunu gösterir.

Tersine $x_{0}, \quad(E-S O P)^{\prime}$ 'nin bir çözümü olsun. $\mathrm{O}$ halde $\forall x \in X /\left\{x_{0}\right\}$ için $T(F(x)) \preccurlyeq_{K}^{m_{1}} T\left(F\left(x_{0}\right)\right)$ olacak şekilde bir $x$ yoktur. Önerme 3.1.'den $\forall x \in X$ için $F(x) \preccurlyeq_{K}^{m_{1}} F\left(x_{0}\right)$ olacak şekilde bir $x$ 'in olmadığı ortaya çıkar. Bu ise $x_{0}$ 'ı $\left(m_{1}-\right.$ $S O P$ )'nin bir çözümü olduğunu verir.

Sonuç 3.4. $F: X \rightrightarrows Y$ kompakt, konveks değerli bir dönüşüm olsun. $x_{0} \in X^{\prime}$ in $\left(m_{1}-\right.$ $S O P$ )'nin bir zayıf çözümü olması için gerek ve yeter koşul $x_{0}$ 'ın

$$
(E-S O P)\left\{\begin{array}{c}
\min (\operatorname{maks}) T(F(x)) \\
x \in X
\end{array}\right.
$$

probleminin bir zayıf çözümü olmasıdır.

Kanıt: Teorem 3.3.'ün kanıtına benzer şekilde Önerme 3.2. kullanılarak elde edilir.

Elde ettiğimiz optimallik koşullarını bir örnek üzerinde uygulayalım.

Örnek 3.4. $F: \mathbb{R}_{+} \rightrightarrows \mathbb{R}^{2}$ kompakt, konveks değerli dönüşümü her $x \in \mathbb{R}_{+}$için $F(x)=$ $\operatorname{conv}\left\{(x, 0),\left(x, x^{2}\right)\right\}$ şeklinde tanımlansın. Sıralama konisi $K=\mathbb{R}_{+}^{2}$ olmak üzere

$$
\left(m_{1}-S O P\right)\left\{\begin{array}{c}
\min F(x) \\
x \in \mathbb{R}_{+}
\end{array}\right.
$$

problemini göz önüne alalım. F'nin bazı görüntü kümeleri Şekil 1. de gösterilmiştir.

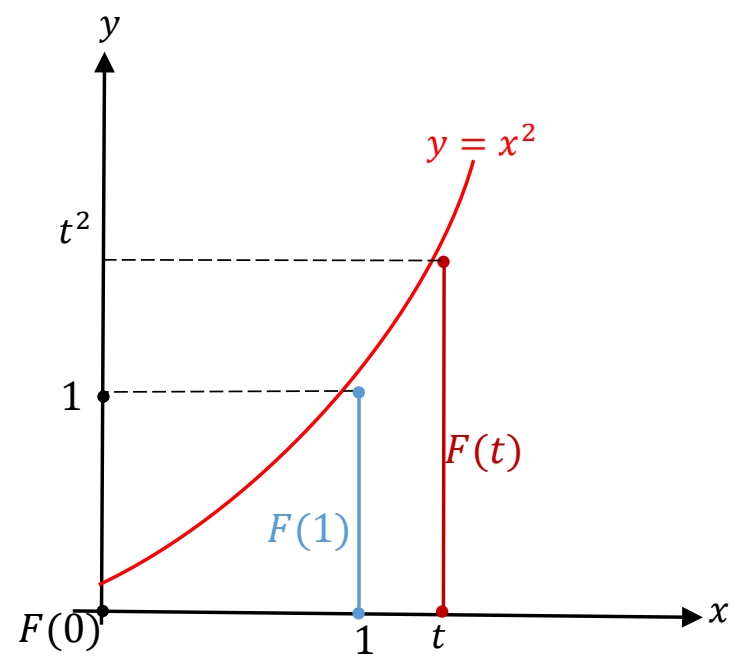

Şekil 1. Örnek 3.4.'deki $F$ küme değerli dönüşümünün bazı görüntü kümeleri

$\forall x \in \mathbb{R}_{+}$için $T(F(x))=[F(x),\{(0,0)\}]$ ve $\mathcal{K}=\left\{[A, B] \mid B \preccurlyeq_{K}^{m_{1}} A\right\}$ olmak üzere,

$$
(E-S O P)\left\{\begin{array}{c}
\min T(F(x)) \\
x \in \mathbb{R}_{+}
\end{array}\right.
$$


problemini ele alalım. $\forall x \in \mathbb{R}_{+}$için $T(F(0)) \preccurlyeq_{\mathcal{K}} T(F(x))$ 'dir. Gerçekten,

$$
\begin{aligned}
T(F(x))-T(F(0)) & =[F(x),\{(0,0)\}]-[F(0),\{(0,0)\}] \\
& =[F(x)-F(0),\{(0,0)\}]=[F(x),\{(0,0)\}]
\end{aligned}
$$

elde edilir. $\{(0,0)\} \preccurlyeq_{C}^{m_{1}} F(x)$ olduğundan $[F(x),\{(0,0)\}] \in \mathcal{K}^{\prime}$ dir. Böylece $0,(E-$ $S O P)^{\prime}$ 'nin bir çözümüdür. Dolayısıyla Teorem 3.3.'den 0 aynı zamanda $\left(m_{1}-\right.$ $S O P$ )'nin de bir çözümüdür.

\section{Sonuç ve Yorum}

Küme değerli optimizasyon problemleri son zamanlarda birçok araştırmacının ilgisini çekmektedir. Yapılan çalışmaların çoğu problemi çözmeye yöneliktir. Problemi çözmek için skalerizasyon, vektörizasyon, yönlü türev ve subdiferansiyel gibi kavramlardan yararlanılır. Bu çalışmada bu yöntemlerden farklı olarak Kuroiwa tarafindan ortaya konulan gömme uzayı ve gömme fonksiyonu yardımı ile $\left(m_{1}-S O P\right)$ için optimallik koşullarının elde edilmesi amaçlanmıştır. Gömme fonksiyonu yardımı ile farklı sıralama bağıntılarına göre verilen küme değerli optimizasyon problemlerinin optimallik koşulları elde edilebilir.

\section{Kaynakça}

[1] A. Chinchuluun, P.M. Pardalos, A. Migdalas, L. Pitsoulis, Pareto Optimality, Game Theory and Equilibria. New York, USA: Springer-Verlag, 2008.

[2] A.A. Khan, C. Tammer, C. Zalinescu, Set-Valued Optimization: An Introduction with Applications. Berlin: Springer-Verlag, 2015.

[3] E. Karaman, M. Soyertem, İ. Atasever Güvenç, D. Tozkan, M. Küçük and Y. Küçük, “A Vectorization for nonconvex set-valued optimization," Turk. J. Math., vol. 42, 2018, pp. 1815-1832.

[4] E. Karaman, M. Soyertem, İ. Atasever Güvenç, D. Tozkan, M. Küçük and Y. Küçük, "Partial order relations on family of sets and scalarizations for set optimization," Positivity, vol. 22 (3), 2018, pp. 783-802.

[5] E. Hernandez and L. Rodriguez-Marin, "Nonconvex scalarization in set optimization with set-valued maps," J. Math. Anal. Appl., vol. 325, 2007, pp. 1-18.

[6] D. Kuroiwa, T. Tanaka and T.X.D. Ha, "On cone convexity of set-valued maps," Nonlinear AnalTheor., vol. 30(3), 1997, pp. 1487-1496.

[7] D. Kuroiwa, "The natural criteria in set-valued optimization," RIMS Kokyuroku, vol. 1031, 1998, pp. 85-90.

[8] D. Kuroiwa, “On set-valued optimization," Nonlinear Anal-Theor., vol. 47 (2), 2001, pp. 1395-1400.

[9] J. Jahn, T.X.D. Ha, "New order relations in set optimization,” J. Optimiz. Theory. App., vol. 148, 2011, pp. 209-236.

[10] M. Pilecka, "Optimality conditions in set-valued programming using the set criterion," Thecnical University of Freiberg, vol. 2014 (2), 2014.

[11] D. Kuroiwa, "On derivative of set-valued maps in set optimization," Kyoto University Research Information Repository, vol. 1611, 2008, pp. 51-55.

[12] D. Kuroiwa, "Canonical type DC set optimization," in Proc. 3th Asian Conference on Nonlinear Analysis and Optimization, Matseu, 2012, pp. 197-204.

[13] D. Kuroiwa, "Generalized minimality in set optimization," Set optimization and applications - the state of the art: from set relations to set-valued risk measures, in mathematics \& statistics, A.H. Hamel, F. Heyde, A. Löhne, B. Rudloff, C. Schrage, Ed. Berlin: Springer proceedings, vol. 151, 2015, pp. 293-311.

[14] D. Kuroiwa, "Some duality theorems of set-valued optimization," RIMS Kokyuroku, vol. 1079, 1999, pp. $15-19$.

[15] D. Pallaschke, R. Urbanski, Pairs of Compact Convex Sets. Dordrecht: Kluwer academic publishers, 2002.

[16] R. Schneider, Convex Bodies: The Brunn-Minkowski Theory. Cambridge: Encyclopedia Math. Appl., 1993. 